\title{
Performance Evaluation of Deception System for Deceiving Cyber Adversaries in Adaptive Virtualized Wireless Networks
}

\author{
Danda B. Rawat and Naveen Sapavath \\ Data Science and Cybersecurity Center (DSC ${ }^{2}$ ) \\ EECS Department, Howard University \\ Washington, DC 20059, USA \\ Email: db.rawat@ieee.org
}

\author{
Min Song \\ Dept. of Electrical \& Computer Engineering \\ Stevens Institute of Technology \\ Hoboken, NJ 07030, USA \\ E-mail: min.song@stevens.edu
}

\begin{abstract}
Malicious actions by cyber-adversaries are growing exponentially which makes it difficult to combat cyber-attacks for emerging networked cyber physical systems (CPS) and Internet of Things (IoT). Furthermore, wireless networks - major communication media for most emerging CPS and IoT applications - are highly vulnerable to cyber attacks because of their nature of open communications. In this paper, we evaluate the performance of the cyber deception system to combat cyber adversaries in virtualized wireless networking framework where software defined network (SDN) controller creates mobile virtual network operators (MVNOs) and continuously senses the network, observes the connections and creates deception MVNO to direct cyber adversaries. The deception MVNO can be used to learn about cyber adversaries in terms of their capabilities, intent and how much damage they can do in the system and so on. Thus, the cyber deception can help secure legitimate users from cyber adversaries. Performance of the proposed approach is evaluated with results obtained from Monte Carlo simulations.
\end{abstract}

\section{CCS CONCEPTS}

- Wireless networks • Mobile and wireless security

\section{KEYWORDS}

Cyber deception in wireless networks, wireless virtualization security, deception MVNO, cyber adversaries in virtualized wireless networks.

\section{ACM Reference format:}

Danda B. Rawat, Naveen Sapavath and Min Song. 2019. Performance Evaluation of Deception System for Deceiving Cyber Adversaries in Adaptive Virtualized Wireless Networks. In Proceedings of The Fourth ACM/IEEE Symposium on Edge Computing (ACM/IEEE SEC'19). Washington DC, November 7-9, 2019, 6 pages.

https://doi.org/10.1145/3318216.3363377

Permission to make digital or hard copies of all or part of this work for personal or classroom use is granted without fee provided that copies are not made or distributed for profit or commercial advantage and that copies bear this notice and the full citation on the first page. Copyrights for components of this work owned by others than ACM must be honored. Abstracting with credit is permitted. To copy otherwise, or republish, to post on servers or to redistribute to lists, requires prior specific permission and/or a fee. Request permissions from permissions@acm.org.

SEC '19, November 7-9, 2019, Arlington, VA, USA

(C) 2019 Association for Computing Machinery.

ACM ISBN 978-1-4503-6733-2/19/11 ..\$15.00

https://doi.org/10.1145/3318216.3363377

\section{INTRODUCTION}

Wireless subscriptions are increasing exponentially with the increasing number of smart connected devices for emerging Internet of Things (IoT) and Cyber Physical Systems (CPS) applications $[7,8,10,12]$. IoT and CPS are expected to use variety of communication media. Wireless medium is one of the best media to provide communication for feedback and control in CPS and IoT. Breakthroughs are needed to support massive number of devices with least delay and high reliability for IoT/CPS applications. Traditionally, wireless spectrum/frequencies are allocated and licensed to wireless service providers for a given period of time (typically long time) and given geographic location (typically for the entire country, state or city). This static allocation approach has been proven to be an inefficient way of using frequentness since most of the time frequencies are either idle or underutilized $[7,12]$. This issue has caused an artificial RF spectrum scarcity. To solve this issue, there have been several efforts including dynamic and opportunistic spectrum access by unlicensed wireless users [12] and wireless virtualization by leasing the infrastructure and RF slices of wireless infrastructure providers (WIPs) to mobile virtual network operators (MVNOs) $[7,9,13]$. Wireless virtualization is regarded as a solution to enhance the spectrum utilization and facilitate the wireless connectivity with the high data rate, better coverage and better quality of experience (QoE) for emerging IoT and CPS applications. Wireless virtualization has the flexibility to adapt the network parameters through software defined network (SDN) controllers based on service level agreements (SLAs) between WIPs and MVNOs on the fly to enhance the $\mathrm{QoE}$ of the end users. Like any other wireless networks, virtual wireless networks are vulnerable to different kinds of cyber attacks [11].

In this paper, we evaluate the performance of the cyber deception system to combat cyber adversaries in wireless virtualization framework. SDN based defender continuously senses the network traffic, observes the connections and creates deception MVNO to direct cyber adversaries to deception MVNO and secure legitimate MVNOs and their users from cyber adversaries. For this problem we consider an Attack Model that consists of malicious wireless users to access unauthorized information wirelessly or overwhelm the wireless resources such as computing and controlling units of MVNOs. To combat adversaries, we propose to use deceiv- 


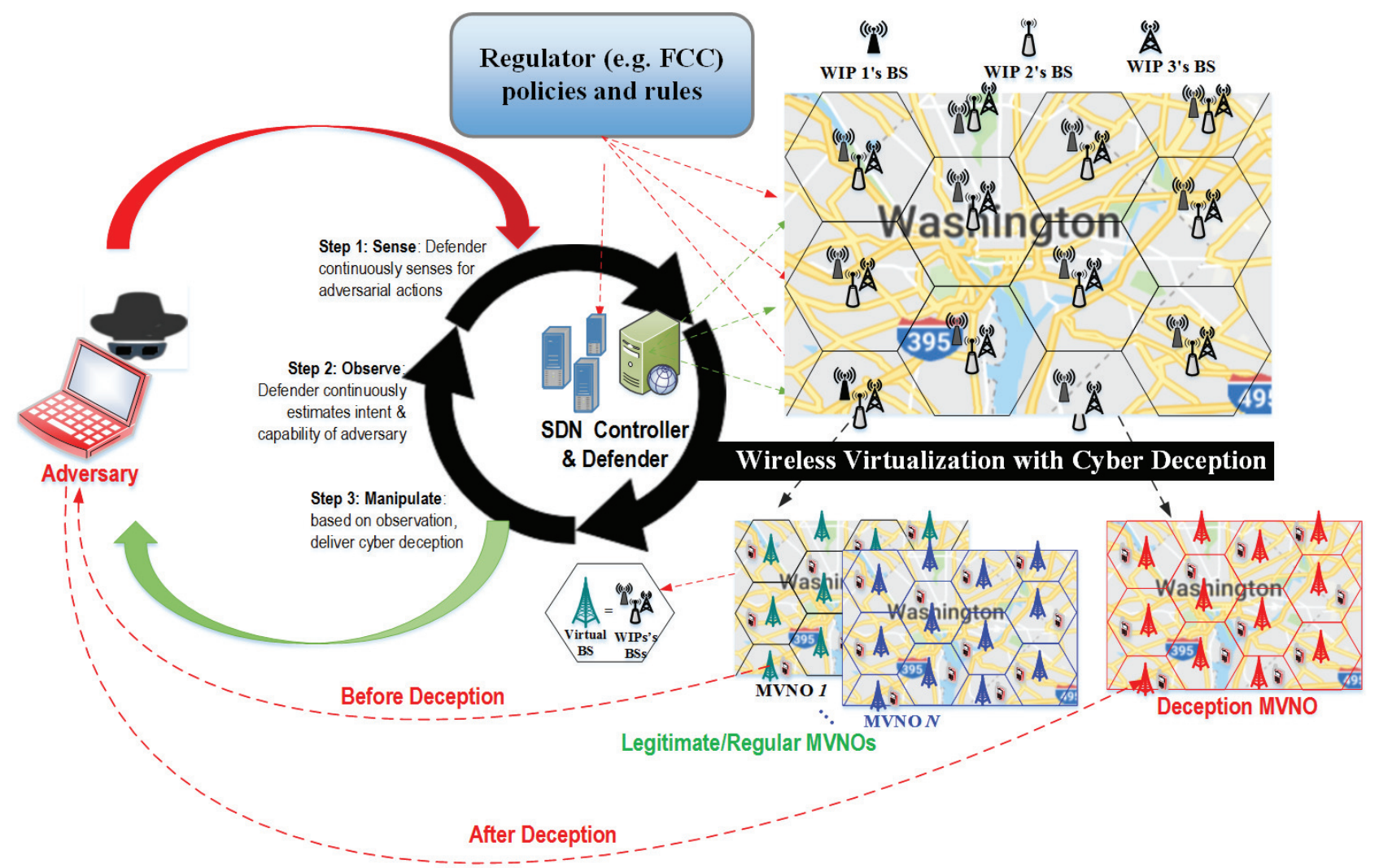

Figure 1: A Typical System Model With an Example Scenario for Deceiving Cyber Adversaries in Wireless Network Virtualization.

ing system to redirect cyber adversaries to deception MVNO so that legitimate wireless users would not suffer from the attack. The performance of the proposed approach is analyzed by using formal mathematical analysis and evaluated by using and numerical results obtained from Monte Carlo simulations.

In the past, several deceptive techniques have been used to solve human conflict. By being motivated from salient features of deception, there have been several effort in digital world and computer networks to combat cyber attacks using cyber deception. The main reason to use deception-based mechanisms is to improve traditional cyber security defenses and learn from attackers using fake system and improve the security of real-system. Recent state-of-the-art works related to cyber deception include $[3,4,6]$. All of these existing approaches deal with cyber deception for traditional network systems. None of the existing works covers the analysis and evaluation of cyber deception in virtual wireless networking framework.

The rest of the paper is organized as follows: Section 2 presents the system model. Section 3 presents the formal analysis followed by the algorithm. Performance evaluation using numerical results is presented in Section 4. Finally, Section 5 concludes the paper.

\section{WIRELESS NETWORK VIRTUALIZATION WITH CYBER DECEPTION SYSTEM}

We consider a typical system model with multiple WIPs who are willing to sublease their RF slices based on their SLAs with MVNOs where multiple MVNOs are created with the help of SDN controller, as shown in Fig. 1. One of the MVNOs will be created as Deception MVNO (DMVNO) to redirect all cyber adversaries. A typical system model with a cyber adversary in wireless network virtualization is depicted in Fig. 1. For cyber deception, SDN controller continuously senses, monitors and observes for cyber adversaries and deploys cyber deception system to redirect the adversaries to DMVNO, as shown in Fig. 1. For the cyber deception system, SDN controller plays a role like a gateway (a media converter) to map the frequency of a given MVNO to the DMVNO for forwarding the adversarial traffic to DMVNO. The approach used for cyber deception is analogous to proxy service or network address translation (NAT) service in the traditional network to provide bridge between adversaries and DMVNO but the adversary would not know how proxy like or NAT like service is being used to redirect it to DMVNO.

In this setup, our goal is to evaluate the performance of the cyber deception system for deceiving cyber- adversaries in the virtual wireless networks. 


\section{ANALYSIS FOR DECEIVING CYBER AD- VERSARIES}

In the virtualized wireless networks, we consider the WIPs sublease their RF slices through SDN controller to MVNOs based on their SLAs. SDN controller helps MVNOs to shrink or grow wireless resources on the fly based on the demand of wireless resources and availability of RF slices. Wireless users subscribe to MVNOs and get wireless services. End users do not see who is serving WIP to their service as MVNOs act like wireless service providers without owning any wireless infrastructure. Furthermore, we consider that the arrival rate of attacks is $\lambda$ and wireless networks of MVNOs take some time to detect adversaries and report to SDN controller. We consider that the time between detecting the attack and deploying the cyber deception system is $D$ time units. Our goal is to find the expected time that we have to wait to deploy a deception system. We start counting attacks at time 0 and let $A_{1}, A_{2}, \ldots$ are the attack inter-arrival times. Furthermore, let us consider that $M$ be the random variable to count the number of attacks that will come before we deploy the cyber-deception system. This process is modeled as Geometric Distribution of Bernoulli trials for $m=1,2,3, \ldots$ to estimate the time $D$ that should be larger than the inter-arrival time of the new attacks. First, we compute $P[\{M=m\}]$ by considering that the attacks are coming independently as

$$
\begin{aligned}
P[\{M=m\}]= & P\left[\left\{A_{1}<D\right\} \cap\left\{A_{2}<D\right\} \cap \ldots\right. \\
& \left.\cap\left\{A_{m}<D\right\} \cap\left\{A_{m+1} \geq D\right\}\right] \\
= & P\left[\left\{A_{1}<D\right\}\right] P\left[\left\{A_{2}<D\right\}\right] \ldots \\
& P\left[\left\{A_{m}<D\right\}\right] P\left[\left\{A_{m+1} \geq D\right\}\right] \\
= & \left(1-e^{-\lambda D}\right)^{m} e^{-\lambda D} .
\end{aligned}
$$

Then, we can write the probability value for the deceived attacks, for the $M$ as the random variable to count the number of attacks that will come before we deploy the cyberdeception system, as

$$
P[\text { Dcved }]=1-\left(1-e^{-\lambda D}\right)^{m} e^{-\lambda D} .
$$

Next, the expected number of new attacks that can come before we deploy cyber deception is estimated as

$$
\begin{aligned}
E[M] & =\sum_{\forall m} m P[\{M=m\}]=\sum_{\forall m} m\left(1-e^{-\lambda D}\right)^{m} e^{-\lambda D} \\
& =\left(1-e^{-\lambda D}\right) e^{-\lambda D} \sum_{\forall m} m\left(1-e^{-\lambda D}\right)^{m-1} \\
& =\left(1-e^{-\lambda D}\right) e^{-\lambda D} e^{2 \lambda D} \\
& =e^{\lambda D}-1
\end{aligned}
$$

Finally, the expected time needed to deploy cyber deception before any new attacks come can be estimated as

$$
E[M] E[A]=\left(e^{\lambda D}-1\right) \frac{1}{\lambda}=\frac{e^{\lambda D}-1}{\lambda}
$$

Furthermore, attack and defense can be formulated as a game with conflicting interests. To find the convergence to equilibrium in a zero-sum game, minimizing the cumulative loss of the defender is of interest. Defender observes the losses $\beta\left(i, B_{t}\right)$ if he had played strategy $i$ for $i=1, \ldots, N$ with defender's actions $G_{t} \in\{1, \ldots, N\}$ and attacker chooses an action $B_{t}=\{1, \ldots, M\}$. If the defender knew the attacker's actions $\left\{B_{1}, B_{2}, \ldots, B_{n}\right\}$ in advance, the defender could choose its strategies to satisfy

$$
G_{t}=\arg \min _{i=1, \ldots, N} \beta\left(i, B_{t}\right)
$$

invoking a total loss

$$
\sum_{t=1}^{n} \min _{i=1, \ldots, N} \beta\left(i, B_{t}\right) .
$$

Getting a least cumulative loss without knowing the attacker's actions, except in obvious situations, is impossible. Thus, the best outcome that can be achieved is to consider the minimization of the difference between defender's cumulative loss and the cumulative loss of the best constant strategy, that is

$$
\text { Cumulative-Loss }=\sum_{t=1}^{n} \beta\left(G_{t}, B_{t}\right)-\min _{i=1, \ldots, N} \sum_{t=1}^{n} \beta\left(i, B_{t}\right) .
$$

Hannan consistency $[1,2]$ suggests that the above difference grows sub-linearly no matter how the attacker plays, which results in uniqueness and existence of the equilibrium point of the game.

Thus, defender and attacker could consider regret-minimizing strategies. We can assume that both defender and attacker play according to Hannan consistent forecasting strategies $[1,2]$. Specifically, assume that the defender chooses his actions $G_{t}$ such that, regardless of what the attacker does,

$\limsup _{n \rightarrow \infty}\left(\frac{1}{n} \sum_{t=1}^{n} \beta\left(G_{t}, B_{t}\right)-\min _{i=1, \ldots, N} \frac{1}{n} \sum_{t=1}^{n} \beta\left(i, B_{t}\right)\right) \leq \epsilon$

where $\epsilon$ is the loss tolerance limit (with loss caused by attacks).

Note that we have assumed that MVNOs take some time to detect the attacks and report the detected attacks to the SDN controller, which, is not considered in this paper, will be our future research. However, we would like to present probabilistic approach for evaluating the attackers' blocking probability (no blocking to blocking all RF slices) to the legitimate users.

We define MVNOs' spectrum state as the number of RF slices available to their users as $\left\{S_{0}, . . S_{k}, . ., S_{q}\right\}, k=\{0,1, . ., q\}$ where $S_{0}$ represents MVNOs are free of attacks and $S_{q}$ represents all RF slices of all MVNOs are attacked/jammed for blocking or denial of service (DoS) to legitimate users. So if the spectrum state is in $S_{q}$, a legitimate user cannot access the wireless services provided by MVNO that means the legitimate user will be denied the wireless service. Then, we can define the probability $P_{k}$ for a state $k$ where $k<q$ and $P_{q}$ denote the DoS/blocking probability. We can consider the attack arrival and attack deception as 
a birth death process [5] $X(t) \mid t>0$ with birth rates (attack arrival rates) $\lambda_{k} \mid k \in \mathbb{N}$ and death rates $\mu_{k} \mid k \in \mathbb{N}$ (attack deception rate). We are interested to find the probability $P_{k}(t)=\operatorname{Pr}[\{X(t)=k\}]$ that the process is in state $S_{k}$ at $t>0$. For an attack arrival rate as $\lambda$ (that is, attack arrival rate at stage $k$ is $\lambda_{k}$ ) and attack deception (attacks being successfully redirected to DMVNOs) rate $\mu$ (that is, attack deception rate at stage $k$ is $\mu_{k}$ ), there exist four different cases for $P_{k}(t+\Delta)$.

- Case 1: no new attacks came and no existing attacks were directed to DMVNO, then the probability that the process was in state $S_{k}$ at time $t$ and no transition $S_{k} \rightarrow S_{k+1}$ and $S_{k} \rightarrow S_{k-1}$ occurred in $\left(t ; t_{d}\right]$, where $t_{d}=t+\Delta$ and $t>0$ and $1>\Delta>0$, can be calculated as

$$
\begin{aligned}
P_{k \rightarrow k}^{\prime} & =P_{k}(t)\left[1-\lambda_{k} \Delta+p_{2}(\Delta)\right]\left[1-\mu_{k} \Delta+p_{2}(\Delta)\right] \\
& =P_{k}(t)\left[1-\lambda_{k} \Delta-\mu_{k} \Delta\right]+p_{2}(\Delta)
\end{aligned}
$$

where the probability of two or more transitions occurring in the interval from $t$ to $t+\delta$ is $p_{2}(\delta)$

- Case 2: the probability that the wireless systems was in state $S_{k-1}$ at time $t$ and a transition $S_{k-1} \rightarrow S_{k}$ occurred in $\left(t ; t_{d}\right]$ can be written as

$$
P_{k-1 \rightarrow k}^{\prime}=P_{k-1}(t)\left[\Delta \lambda_{k-1}+p_{2}(\Delta)\right]=P_{k-1}(t) \Delta \lambda_{k-1}+p_{2}
$$

- Case 3: the probability that the wireless systems was in state $S_{k+1}$ at time $t$ and a transition $S_{k+1} \rightarrow S_{k}$ occurred in $\left(t ; t_{d}\right]$ can be written as

$$
P_{k+1 \rightarrow k}^{\prime}=P_{k+1}(t)\left[\Delta \mu_{k+1}+p_{2}(\Delta)\right]=P_{k+1}(t) \Delta \mu_{k+1}+p_{2}(\Delta)
$$

- Case 4: the probability that the two or more transitions occurred in $(t ; t+\Delta]$ which leave the process in state $S_{k}$ is given by

$$
P_{k \pm z \rightarrow k}^{\prime}=p_{2}(\Delta), \quad z \geq 2
$$

Since these events are mutually exclusive, it follows that for $n>0$ (the DoS/blocking probability by occupying $k$ states)

$$
\begin{aligned}
P_{k}(t+\Delta)= & {\left[1-\lambda_{k} \Delta-\mu_{k} \Delta\right] P_{k}(t)+\Delta \lambda_{k-1} P_{k-1}(t)+} \\
& +\Delta \mu_{k+1} P_{k+1}(t)+p_{2}(\Delta)
\end{aligned}
$$

A similar reasoning yields, in case $n=0$

$$
P_{0}(t+\Delta)=\left[1-\lambda_{0} \Delta\right] P_{0}(t)+\Delta \mu_{1} P_{1}(t)+p_{2}(\Delta)
$$

\subsection{The Algorithm}

Based on the analysis above, the formal algorithmic steps are presented as Algorithm 1.
Algorithm 1: Evaluation of Cyber Deception System in Wireless Virtualization

Output: \# of attacks to MVNO, \# of attacks to MVNO after deceiving, expected attack time

Input: $\lambda$ values (say $\lambda=0.4 .0 .6,0.8$ per sec), attack tolerance limit $\epsilon$, and deception time to redirect traffic to DMVNO;

while $M V N O$ s are operating AND value in eq. (5) $\leq \epsilon$ do

Estimate the number of attacks at MVNOs using equation (3);

Estimate the number of attacks deceived/redirected to DMVNO using (2);

Estimate the time needed to deceive/redirect the attack to DMVNO using equation (4);

Estimate the probability value from (10) or (11) depending on the cases;

end

The time complexity of the algorithm is in the order of $\mathcal{O}(Y Z)$ where $Y$ denotes the total number of legitimate MVNOs that are running deception system and $Z$ denotes the total number of attacks in the system.

\section{PERFORMANCE EVALUATION}

In order to evaluate the performance of deployment of cyber deception system, we have used extensive simulations by considering MVNOs, a DMVNO, legitimate wireless users for MVNOs and the attackers. First, we have plotted the variation for the number of attacks vs. the cyber deception deployment time, as shown in Fig. 2. We observed that taking a longer time for cyber deception deployment results in more number of attacks to the MVNO for a given attackarrival rate, as shown in Fig. 2. For instance, when the $\Delta$ ) deception deployment time is 4 seconds with attack arrival rates $0.4,0.6$ and 0.8 per second, the number of attacks get doubled 5, 10, 22 attacks, respectively, as shown in Fig. 2. Furthermore, when the arrival rate is high, say $\lambda=0.8$, with increasing deployment time, exponentially increasing number of attacks/attackers are observed at MVNOs.

Next, we plotted the variation of the number of attacks deceived (redirected to DMVNO) vs the deception deployment time, as shown in Fig. 3 where we observed that earlier cyber deception deployment results in more deceived attacks (attacks redirected from MVNOs to DMVNO) for a given attack arrival rate. Furthermore, higher attack arrival rate results in more attacks for a given deception deployment time, as shown in Fig. 3. For instance, when the deception deployment time is 5 seconds with attack arrival rates 0.4 , 0.6 and 0.8 per second, number of deceived attacks from MVNOs' side get to zero, as shown in Fig. 3. In other words, all attacks were redirected to DMVNO and no attacks were hampering legitimate MVNOs because of deception, as shown in Fig. 3.

Then, we plotted the variation of expected time for MVNO being attacked (aka, expected waiting time for the attack to be deceived to DMVNO) vs the deception deployment time for different attack arrival rates, as shown in Fig. 4. More time we take to deploy deception, attackers will be there for a longer time and attack the MVNOs before deceiving them 
to DMVNO, as shown in Fig. 4. For instance, when the deception deployment time is 4 seconds with attack arrival rates $0.4,0.6$ and 0.8 per second, expected attack time duration increases from 10 seconds to 18 seconds to 30 seconds, as shown in Fig. 4. Furthermore, when the arrival rate is high, say $\lambda=0.8$ attacks/sec, with increasing deployment time, the total time duration for attacks gets exponentially increased for MVNOs, as shown in Fig. 4.

Finally, we plotted variation of attack arrival rate, deception rate and successful attack rate after deploying deception vs the time, as shown in Fig. 5. We observed that when deception rate is lower (or higher) than attack arrival rate, the system will have (or not have) attacks and their impact, as shown in Fig. 5.

In summary, with the successful deployment of cyber deception system, the attack impact of the cyber adversaries can be significantly reduced for legitimate MVNOs. Deployment of cyber deception system should be quick enough to handle given attack arrival rates to minimize the attack impact on the network.

\section{CONCLUSION}

This paper has presented the performance evaluation of cyber deception system to combat cyber adversaries in virtualized wireless networks using a probabilistic approach. We have proposed to leverage the dynamic nature of wireless virtualization where SDN controller helps to form MVNOs and DMVNO using WIPs' wireless resources on the fly based on the SLAs. The SDN controller continuously senses the network, observes the connections and redirects cyber adversaries to DMVNO. Cyber adversaries capabilities, intent

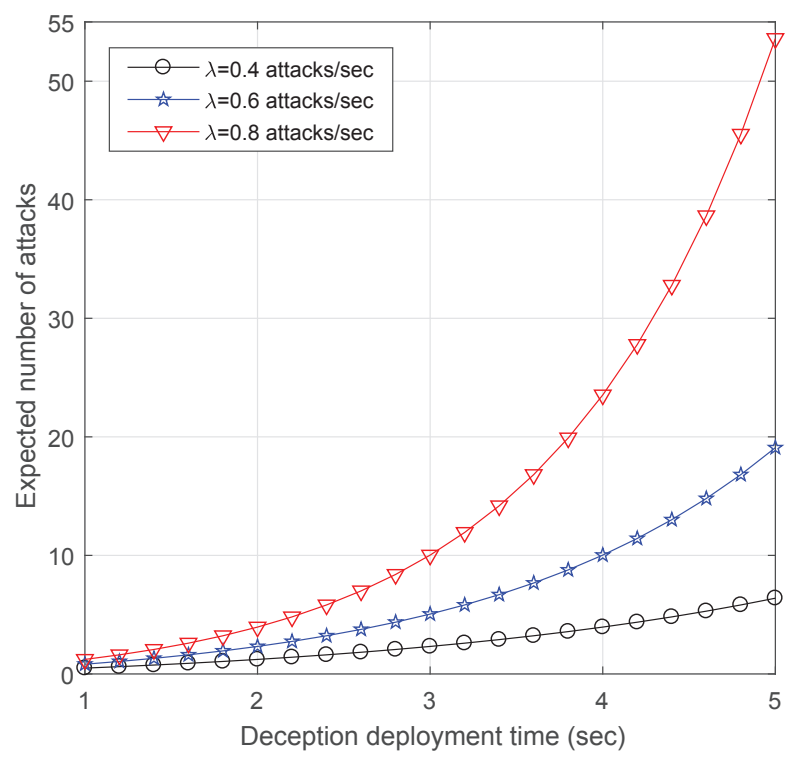

Figure 2: Number of attacks vs deception deployment time for deceiving cyber adversaries in virtualized wireless networks.

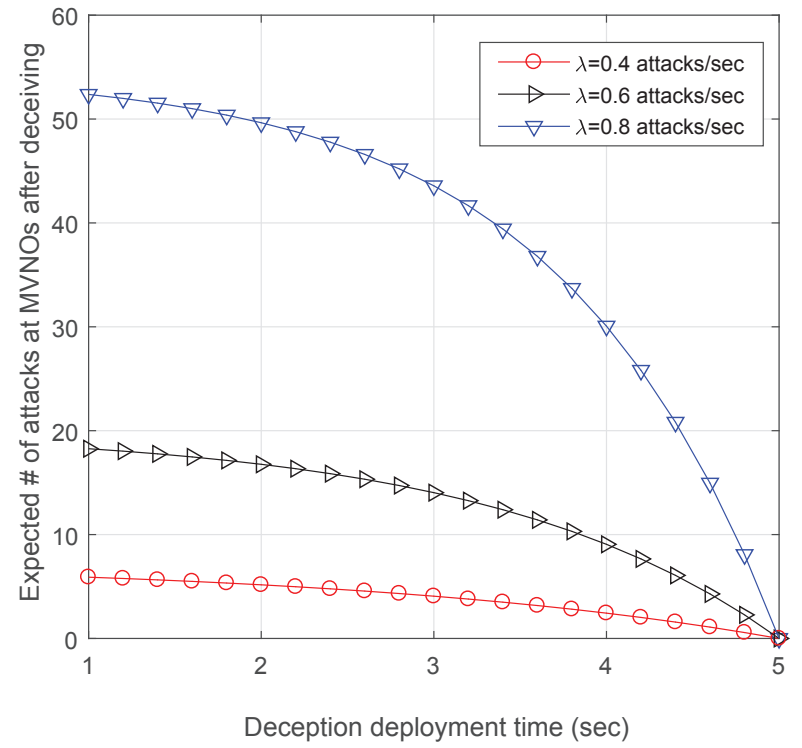

Figure 3: Number of attacks deceived (from MVNOs' side) vs deception deployment time for deceiving cyber adversaries in virtualized wireless networks.

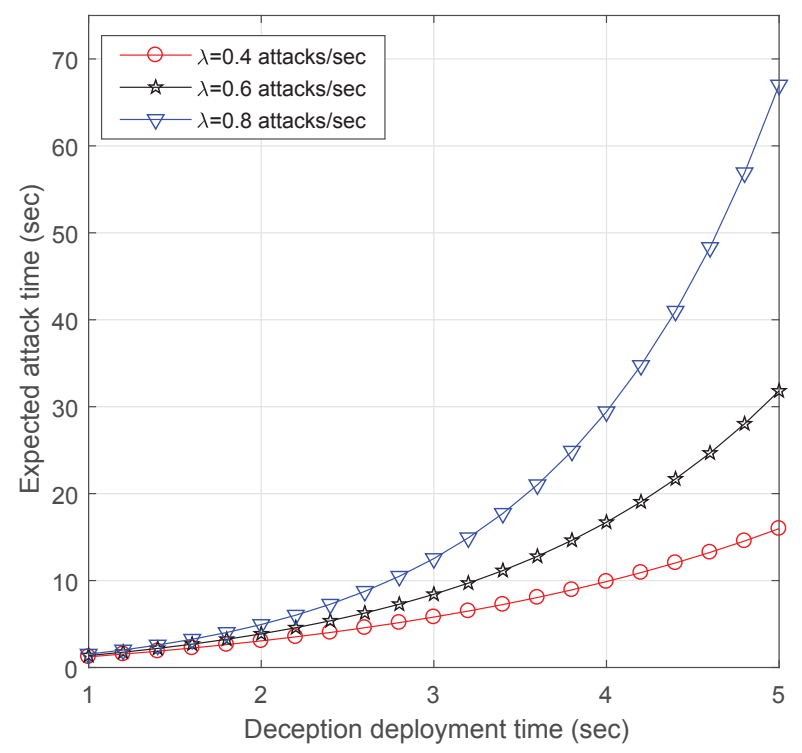

Figure 4: Successful attack time (expected waiting time for the attack to be deceived to DMVNO) vs deception deployment time for deceiving cyber adversaries in virtualized wireless networks.

and how much damage they can do in the system can be obtained through DMVNO. We observed that, for a given arrival rate of attacks, sooner deployment of cyber deception not only helps to reduce number of attacks in MVNOs 


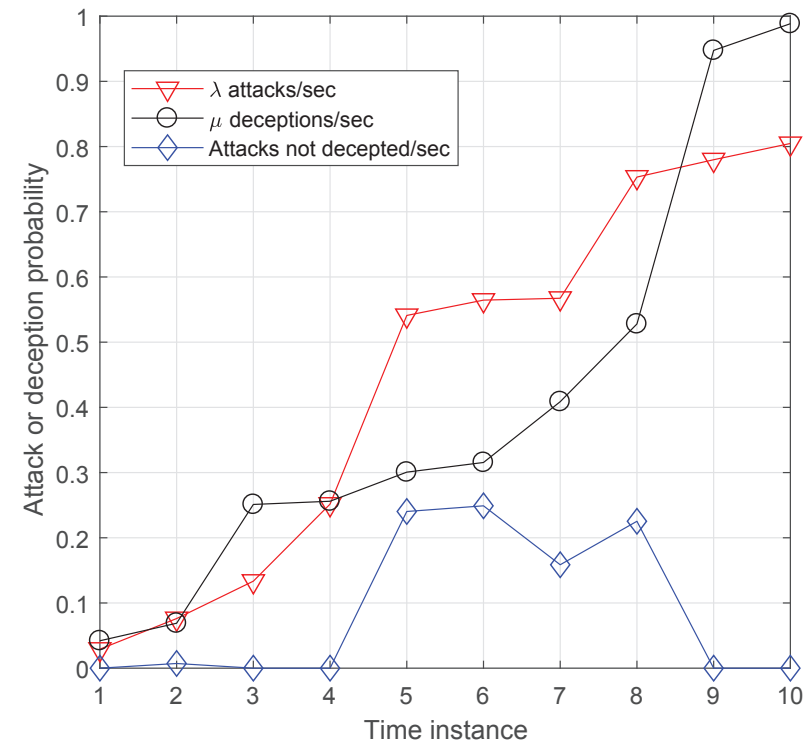

Figure 5: Variation of attack arrival rate, deception rate and successful attack rate after deploying deception vs the time.

but also helps to increase the deception of attacks in virtual wireless networks.

\section{Acknowledgments}

This work is partly supported by the U.S. National Science Foundation (NSF) under grants CNS 1650831 and the U.S. Department of Homeland Security under grant DHS 2017ST-062-000003. However, any opinion, finding, and conclusions or recommendations expressed in this document are those of the authors and should not be interpreted as necessarily representing the official policies, either expressed or implied, of the funding agencies.

\section{REFERENCES}

[1] Allenberg, C., Auer, P., Györfi, L., And OTTUCSÁk, G. Hannan consistency in on-line learning in case of unbounded losses under partial monitoring. In International Conference on Algorithmic Learning Theory (2006), Springer, pp. 229-243.

[2] Cesa-Bianchi, N., And Lugosi, G. Prediction, learning, and games. Cambridge university press, 2006.

[3] Chiang, C.-Y. J., Gottlieb, Y. M., Sugrim, S. J., Chadha, R., Serban, C., Poylisher, A., Marvel, L. M., And SAntos, J. ACyDS: An adaptive cyber deception system. In MILCOM 2016-2016 IEEE Military Communications Conference (2016), pp. $800-805$.

[4] Jajodia, S., Subrahmanian, V., Swarup, V., And WANG, C. Cyber Deception. Springer, 2016.

[5] Kendall, D. G., ET Al. On the generalized" birth-and-death" process. The annals of mathematical statistics 19, 1 (1948), 1-15.
[6] Kumar, A. A., Mohan, A. K., And Amritha, P. Deceiving attackers in wireless local area networks using decoys. Journal of Cyber Security and Mobility 7, 1 (2018), 201-214.

[7] Liang, C., AND Yu, F. R. Wireless network virtualization: A survey, some research issues and challenges. IEEE Communications Surveys 8 Tutorials 17, 1 (2015), 358-380.

[8] Rawat, D. B., Alshaikhi, A., Alshammari, A., Bajracharya, C., And Song, M. Payoff optimization through wireless network virtualization for IoT applications: A three layer game approach. IEEE Internet of Things Journal 6, 2 (2018), 2797-2805.

[9] Rawat, D. B., Alsulami, B. S., And Chaudhary, V. On the wireless virtualization with QoE constraints. Wiley's Transactions on Emerging Telecommunications Technologies 30, 3 (2019), e3573.

[10] Rawat, D. B., Rodrigues, J. J., AND Stojmenovic, I. Cyber-physical systems: from theory to practice. CRC Press, 2015.

[11] Rawat, D. B., AND Sharma, N. Wireless Network Virtualization for Enhancing Security: Status, Challenges and Perspectives. In Proc. of the IEEE SoutheastCon 2016 (2016), pp. 1-8.

[12] Rawat, D. B., Song, M., And Shetty, S. Dynamic spectrum access for wireless networks. Springer, 2015.

[13] Wang, X., Krishnamurthy, P., And Tipper, D. Wireless Network Virtualization. In Proc. of the IEEE 2013 International Conference on Computing, Networking and Communications (ICNC) (2013), pp. $818-822$. 